\title{
Tissue discrimination in anterior eye using three optical parameters obtained by polarization sensitive optical coherence tomography
}

\author{
Arata Miyazawa $^{1,5}$, Masahiro Yamanari ${ }^{1,5}$, Shuichi Makita ${ }^{1,5}$, \\ Masahiro Miura $^{2,5}$, Keisuke Kawana ${ }^{3,5}$, Keiichi Iwaya ${ }^{2,4}$, \\ Hiroshi Goto ${ }^{2}$ and Yoshiaki Yasuno ${ }^{1,5}$ \\ ${ }^{1}$ Computational Optics Group in the University of Tsukuba, Ibaraki, Japan, \\ ${ }^{2}$ Tokyo Medical University, Tokyo, Japan, \\ ${ }^{3}$ Department of Ophthalmology, Institute of Clinical Medicine, University of Tsukuba, Ibaraki, \\ Japan, \\ ${ }^{4}$ Department of Pathology, National Defense Medical College, Saitama, Japan, \\ ${ }^{5}$ Computational Optics and Ophthalmology Group, Tsukuba, Ibaraki, Japan \\ yasuno@optlab2.bk.tsukuba.ac.jp
}

http://optics.bk.tsukuba.ac.jp/COG/

\begin{abstract}
We developed a tissue discrimination algorithm of polarization sensitive optical coherence tomography (PS-OCT) based on the optical properties of tissues. We calculated the three-dimensional (3D) feature vector from the parameters intensity, extinction coefficient, birefringence, which were obtained by PS-OCT. The tissue type of each pixel was determined according to the position of the feature vector in the 3D feature space. The algorithm was applied for discriminating tissues of the human anterior eye segment. The conjunctiva, sclera, trabecular meshwork (TM), cornea, and uvea were well separated in the 3D feature space, and we observed them with good contrast. The TM line can be observed in the $3 \mathrm{D}$ discriminated volume, as observed by gonioscopy. We validated our method by applying our algorithm and histological data to porcine eyes. A marker was injected into sub-Tenon's space and the tissues that were anterior to the marker and posterior to the marker were successfully segmented by our algorithm.
\end{abstract}

(C) 2009 Optical Society of America

OCIS codes: (100.5010) Pattern recognition and feature extraction; (170.4470) Ophthalmology; (170.4500) Optical coherence tomography; (230.5440) Polarization sensitive devices.

\section{References and links}

1. D. Huang, E. A. Swanson, C. Lin, J. Schuman, W. Stinson, W. Chang, M. R. Hee, T. Flotte, K. Gregory, C. Puliafito, and J. Fujimoto, "Optical coherence tomography," Science 254, 1178-1181 (1991).

2. T. Gambichler, G. Moussa, M. Sand, D. Sand, P. Altmeyer, and K. Hoffmann, "Applications of optical coherence tomography in dermatology," J. Dermatl. Sci. 40, 85-94 (2005).

3. S. A. Boppart, B. E. Bouma, C. Pitris, G. J. Tearney, J. F. Southern, M. E. Brezinski, and J. G. Fujimoto, "Intraoperative assessment of microsurgery with three-dimensional optical coherence tomography," Radiology 208 , 81-86 (1998). 
4. V. Westphal, A. M. Rollins, J. Willis, M. V. Sivak Jr, and J. A. Izatt, "Correlation of endoscopic optical coherence tomography with histology in the lower-GI tract," Gastrointest. Endosc. 61, 537-546 (2005).

5. A. M. Sergeev, V. M. Gelikonov, G. V. Gelikonov, F. I. Feldchtein, R. V. Kuranov, and N. D. Gladkova, "In vivo endoscopic OCT imaging of precancer and cancer states of human mucosa,” Opt. Express 1, 432-440 (1997), http://www.opticsexpress.org/abstract.cfm?URI=oe-1-13-432.

6. A. V. D‘Amico, M. Weinstein, X. Li, J. P. Richie, and J. G. Fujimoto, "Optical coherence tomography as a method for identifying benign and malignant microscopic structures in the prostate gland," Urology 55, 783-787 (2000).

7. K. W. Gossage, T. S. Tkaczyk, J. J. Rodriguez, and J. K. Barton, “Texture analysis of optical coherence tomography images: feasibility for tissue classification,” J. Biomed. Opt. 8, 570-575 (2003).

8. X. Qi, M. V. Sivak Jr, G. Isenberg, J. E. Willis, and A. M. Rollins, "Computer-aided diagnosis of dysplasia in Barrett's esophagus using endoscopic optical coherence tomography,” J. Biomed. Opt. 11, 044010 (2006).

9. C. A. Lingley-Papadopoulos, M. H. Loew, M. J. Manyak, and J. M. Zara, "Computer recognition of cancer in the urinary bladder using optical coherence tomography and texture analysis," J. Biomed. Opt. 13, 024003 (2008)

10. A. M. Zysk and S. A. Boppart, "Computational methods for analysis of human breast tumor tissue in optical coherence tomography images," J. Biomed. Opt. 11, 054015 (2006).

11. H. Ishikawa, D. M. Stein, G. Wollstein, S. Beaton, J. G. Fujimoto, and J. S. Schuman, "Macular segmentation with optical coherence tomograpy," Invest. Ophthalmol. Visual Sci. 46, 2012-2016 (2005).

12. M. Mujat, R. C. Chan, B. Cence, B. H. Park, C. Joo, T. Akkin, T. C. Chen, and J.F. de Boer, "Retinal nerve fiber layer thickness map determined from optical coherence tomography images," Opt. Express 13, 9480-9491 (2005), http://www.opticsexpress.org/abstract.cfm?URI=oe-13-23-9480.

13. D. C. Fernández, H. M. Slinas, and C. A. Puliafito, "Automated detection of retinal layer structures on optical coherence tomography images," Opt. Express 13, 10200-10216 (2005), http://www.opticsexpress.org/abstract.cfm?URI=oe-13-25-10200.

14. M. Szkulmowski, B. Sikorski, T. Bajraszewski, V. J. Srinivasan, A. Szkulmowska, J. J. Kaluzny, J. G. Fujimoto, and A. Kowalczyk, "Analysis of posterior retinal layers in spectral optical coherence tomography images of the normal retina and retinal pathologies," J. Biomed. Opt. 12, 041207 (2007).

15. Y. Yasuno, V. D. Madjarova, S. Makita, M. Akiba, A. Morosawa, C. Chong, T. Sakai, K. Chan, M. Itoh, and T. Yatagai, "Three-dimensional and high-speed swept-source optical coherence tomography for in vivo investigation of human anterior eye segments," Opt. Express 13, 10652-10664 (2005), http://www.opticsexpress.org/abstract.cfm?URI=oe-13-26-10652.

16. L. M. Sakata, R. Lavanya, D. S. Friedman, H. T. Aung, S. K. Seah, P. J. Foster, and T. Aung, "Assessment of the scleral spur in anterior segment optical coherence tomography," Arch. Ophthalmol. 126, 181-185 (2008).

17. S. Asrani, M. Sarunic, C. Santiago, and J. Izatt, "Detailed visualization of the anterior segment using Fourierdomain optical coherence tomography," Arch. Ophthalmol. 126, 765-771 (2008).

18. Y. Yasuno, M. Yamanari, K. Kawana, T. Oshika, and M. Miura, "Investigation of post-glaucoma-surgery structures by three-dimensional and polarization sensitive anterior eye segment optical coherence tomography," Opt. Express 17, 3980-3996 (2009), http://www.opticsexpress.org/abstract.cfm?URI=oe-17-5-3980.

19. K. Kawana, T. Kiuchi, Y. Yasuno, and T. Oshika, "Evaluation of trabeculectomy blebs using 3-dimensional cornea and anterior segment optical coherence tomography," Ophthalmol. 116, 848-855 (2009).

20. C. Xu, J. M. Schmitt, S. G. Carlier, and R. Virmani, "Characterization of atherosclerosis plaques by measuring both backscattering and attenuation coefficients in optical coherence tomography," J. Biomed. Opt. 13, 034003 (2008).

21. R. Duda, P. Hart, and D. Stork, Pattern Classification, 2nd ed (Wiley, New York, 2001).

22. M. Mujat, R. D. Ferguson, D. X. Hammer, C. Gittins, and N. Iftimia, "Automated algorithm for breast tissue differentiation in optical coherence tomography," J. Biomed. Opt. 14, 034040 (2009).

23. E. Götzinger, M. Pircher, W. Geitzenauer, C. Ahlers, B. Baumann, S. Michels, U. Schmidt-Erfurth, and C. K. Hitzenberger, "Retinal pigment epithelium segmentation by polarization sensitive optical coherence tomography," Opt. Express 16, 16410-16422 (2008), http://www.opticsexpress.org/abstract.cfm?URI=oe-16-21-16410.

24. M. R. Hee, D. Huang, E. A. Swanson, and J. G. Fujimoto, "Polarization-sensitive low-coherence reflectometer for birefringence characterization and ranging,” J. Opt. Soc. Am. B 9, 903-908 (1992).

25. J. F. de Boer, T. E. Milner, M. J. C. van Gemert, and J. S. Nelson, "Two-dimensional birefringence imaging in biological tissue by polarization-sensitive optical coherence tomography," Opt. Lett. 22, 934-936 (1997).

26. C. K. Hitzenberger, E. Götzinger, M. Sticker, M. Pircher, and A. F. Fercher, "Measurement and imaging of birefringence and optic axis orientation by phase resolved polarization sensitive optical coherence tomography," Opt. Express 9, 780-790 (2001), http://www.opticsexpress.org/abstract.cfm?URI=oe-9-13-780.

27. B. H. Park, M. C. Pierce, B. Cense, and J. F. de Boer, "Jones matrix analysis for a polarization-sensitive optical coherence tomography system using fiber-optic components," Opt. Lett. 29, 2512-2514 (2004).

28. M. Todorovic, S. L. Jial, and L. V. Wang, "Determination of local polarization properties of biological samples in the presence of diattenuation by use of Mueller optical coherence tomography," Opt. Lett. 29, 2402-2404 (2004)

29. N. J. Kemp, H. N. Zaatari, J. Park, H. G. Rylander, and T. E. Milner, "Form-biattenuance in fibrous tissues measured with polarization-sensitive optical coherence tomography (PS-OCT)," Opt. Express 13, 4611-4628 
(2005), http://www.opticsexpress.org/abstract.cfm?URI=oe-13-12-4611.

30. B. H. Park, C. Saxer, S. M. Srinivas, J. S. Nelson, and J. F. de Boer, "In vivo burn depth determination by highspeed fiber-based polarization sensitive optical coherence tomography," J. Biomed. Opt. 6, 474-479 (2001).

31. B. Cense, T. C. Chen, B. H. Park, M. C. Pierce, and J. F. de Boer, "Thickness and birefringence of healthy retinal nerve fiber layer tissue measured with polarization-sensitive optical coherence tomography," Invest. Ophthalmol. Visual Sci. 45, 2606-2612 (2004).

32. M. Pircher, E. Götzinger, R. Leitgeb, and C. K. Hitzenberger, "Transversal phase resolved polarization sensitive optical coherence tomography," Phys. Med. Biol. 49, 1257-1263 (2004).

33. M. Yamanari, S. Makita, and Y. Yasuno, "Polarization-sensitive swept-source optical coherence tomography with continuous source polarization modulation," Opt. Express 16, 5892-5906 (2008), http://www.opticsexpress.org/abstract.cfm?URI=oe-16-8-5892.

34. S. Makita, M. Yamanari, and Y. Yasuno, "In vivo depth-resolved tissue contrast by local birefringence and differential optic axis orientation using polarization-sensitive swept-source optical coherence tomography," Proc. SPIE 7168, 716888 (2009).

35. M. Yamanari, S. Makita, V.D. Madjarova, T. Yatagai, and Y. Yasuno, "Fiber-Based Polarization-Sensitive Fourier Domain Optical Coherence Tomography using B-Scan-Oriented Polarization Modulation Method,” Opt. Express 14, 6502-6515 (2006), http://www.opticsinfobase.org/abstract.cfm?URI=oe-14-14-6502.

36. M. Yamanari, Y. Lim, S. Makita, and Y. Yasuno, "Visualization of phase retardation of deep posterior eye bypolarization-sensitive swept-sourceoptical coherence tomography with1-textmum probe," Opt. Express 17, 12385-12396 (2009), http://www.opticsexpress.org/abstract.cfm?URI=oe-17-15-12385.

37. M. Yamanari, M. Miura, S. Makita, T. Yatagai, and Y. Yasuno, "Phase retardation measurement of retinal nerve fiber layer by polarization-sensitive spectral-domain optical coherence tomography and scanning laser polarimetry,” J. Biomed. Opt. 13, 014013 (2008).

\section{Introduction}

Optical coherence tomography (OCT) can be used to obtain information on the cross-sectional structure of biological samples [1]. OCT uses partially coherent near-infrared light and detects backscattered light from the sample with the help of a low-coherence interferometer. Because OCT has the potential to produce high-resolution images at near-video rates, it is expected to be used for the diagnosis of diseases.

OCT has been used to identify the tissue types of highly scattering biological structures such as the skin [2], vascular tissue [3], gastrointestinal tract [4], urinary bladder [5], and prostate [6]. In these cases, identification is based on the structural information, e.g., the presence or absence of structures and layers as visualized by the scattering intensity.

On the other hand, many normal tissues, such as the sclera and aorta, show few structures in OCT images and classification of these tissues seems to be difficult. When visible structural features cannot be observed, texture analysis is useful for distinguishing between tissue types. Texture analysis was first used for classifying normal and abnormal mouse tissues [7]. Texture analysis has been applied for the diagnosis of dysplasia in the esophagus [8] and cancer in the urinary bladder [9]. Moreover, breast cancer has also been diagnosed by image analysis [10].

These reports suggest that the difference in the optical properties of tissue types are well acquired by OCT, even when no visible structure can be observed in the scattering OCT image.

In the field of ophthalmology, a levelset segmentation algorithm based on structural information was developed for the posterior eye, and the retinal layers were segmented [11-14]. Segmentation of retinal layers is clinically important because quantitative measurement of their thickness may be used to diagnose diseases. For instance, the thickness of the retinal nerve fiber layer is related to glaucoma, and the thickness of the retinal pigment epithelium (RPE) is related to age related macular degeneration.

However, segmentation based on structural information of the anterior eye is difficult because the scattering properties of tissues in the anterior eye are not so different [15-19]. However, there is a clinical demand for the differentiation of the trabecular meshwork (TM) from other tissues in the anterior eye. The TM is a drainage of the aqueous humor of the anterior eye chamber and controls intraocular pressure. If drainage from the TM is blocked, the pressure in 
the eye, i.e., the intraocular pressure, increases, resulting in angle-closure glaucoma. Hence, the position of the TM can be used as a landmark for screening patients for the risk of angle-closure glaucoma. Therefore, we attempted to develop an algorithm for differentiating between tissues in the anterior eye on the basis of their optical properties and not structural information.

There were some reports on tissue discrimination based on optical parameters obtained by OCT. $\mathrm{Xu}$ et al. characterized 3 types of atherosclerosis plaques in coronary arteries was performed by analysis of 2 optical parameters obtained by OCT [20]. The results of this report indicate that a combination of 2 parameters offers better discrimination; moreover, it is well known that analysis in a higher dimensional feature space can increase the accuracy of segmentation [21].

In the report of Mujat et al., differentiating of 3 breast tissue types was demonstrated by analysis of 8 parameters obtained by OCT [22]. This report also indicate that analysis in a higher dimensional space is expected to produce better classification.

Segmentation of the RPE was performed by Götzinger et al. by using optical parameters obtained by polarization sensitive-OCT (PS-OCT) [23]. PS-OCT is an extension of conventional OCT, which can measure birefringence (phase retardation, optic axis, and diattenuation [24-29]) in addition to backscattering intensity. RPE segmentation was performed by the broad distribution of retardation values caused by polarization scrambling at the RPE. We think that the birefringence measured by PS-OCT is also useful for tissue discrimination in the anterior eye. Because of the increase in phase retardation along the depth, which indicates birefringence [30,31], collagen fibrous tissue of the sclera and TM can be observed in the phaseretardation image of the anterior eye [32,33].

In this paper, we demonstrate an algorithm for discriminating between tissues in the anterior eye and create a clinically useful image for angle assessment related to glaucoma. With this aim, we used the results of the previous reports. That is, we calculated the optical parameters of tissues based on both intensity and birefringence as measured by PS-OCT and conducted the analysis by using a combination of 3 optical parameters.

\section{Method}

We measured 4 eyes of 4 subjects by PS-OCT and applied the following discrimination algorithm. We calculated 3 optical property values that were measured by PS-OCT. These properties were used as features of the tissue being analyzed. Hence, each pixel has a set of 3 feature values, which are together referred to as the three-dimensional (3D) feature vector. If adequate features are used, the distributions of the feature vectors of different tissues will be separated in a corresponding 3D feature space. All pixels can be discriminated to identify the types of tissues according to the positions of the feature vectors in the feature space.

\subsection{PS-OCT system}

The OCT system employed in this study was polarization sensitive swept source OCT (PSSS-OCT) with source polarization modulation [33]. This system can be used to calculate OCT intensity, diattenuation, phase retardation, and relative optic axis orientation of the sample on the basis of the measured Jones matrix. The center wavelength of the light source was $1.31 \mu \mathrm{m}$, and the scanning rate was 20,000 A-lines/s. The axial and lateral resolutions were $11.9 \mu \mathrm{m}$ and $32.3 \mu \mathrm{m}$, respectively. The image size of the B-scan was 605 (axial) $\times 512$ (lateral) pixels, obtained from the measurement range of $4 \times 5 \mathrm{~mm}$.

The examination protocol of PS-OCT was designed to adhere to the tenets of the Declaration of Helsinki and was approved by the institutional review boards of the University of Tsukuba. 


\subsection{Features of the tissue being studied}

The parameters used in this study were backscattered intensity (I), extinction coefficient (EC), and birefringence (BR).

The backscattered intensity with a linear scale, which was calculated as the sum of the intensities of horizontally and vertically polarized zeroth-order OCT signals [33], was used as a feature of the tissues (I).

The extinction coefficient is calculated as the slope of the logarithmic intensity along the depth (EC). This slope can be considered as a sum of the scattering coefficient, absorption coefficient, and systematic OCT signal decay. Since the decay is the same for each A-scan, this coefficient is regarded as an optical property of tissues. The least squares fitting is applied to the logarithmic intensity to obtain the slope with a kernel size of 45 pixels $(297 \mu \mathrm{m})$.

Although phase-retardation OCT images show the presence of birefringence as the increase of retardation along the depth, the raw phase retardation could not be used as a feature parameter. This is because retardation measured by PS-OCT is cumulative along the depth, which means that birefringence is not indicated by the value of retardation, but by the increase ratio of retardation along the depth. Additionally, the range of retardation is limited to 180 degrees because of phase wrapping. Therefore, we calculated local birefringence based on Jones matrix [34] with a kernel size of 14 pixels $(93 \mu \mathrm{m})$ and used it as a feature of the tissues (BR).

For reducing the noise effect of speckle, the mean value of pixels in a moving window (size: $15 \times 15$ pix $=99 \mu \mathrm{m}$ (axial) $\times 146 \mu \mathrm{m}$ (lateral)) was calculated as the value of each feature (I, $\mathrm{EC}$, and $\mathrm{BR}$ ) and used for analysis.

\subsection{Tissue discrimination}

The region that can be considered to be a part of a target tissue is selected manually as a reference region. We set the following 5 target tissues and selected 5 reference regions for each: the conjunctiva, sclera, TM, cornea, and uvea. This selection was based on the anatomical and histological features of the tissues. The combination of 3 features, i.e., the 3D feature vector, was plotted in the 3D feature space. Then, from these plots, we created similarity distribution maps for each reference region. These similarity distribution maps were calculated as the sum of Gaussian kernels in order to ensure that the distributions in the feature space were smooth and continuous. Namely, the similarity distribution map of the $i$-th reference region is defined as

$$
D_{i}(\mathbf{x}) \equiv \sum_{j=0}^{M_{i}-1} G\left(\mathbf{x}-\boldsymbol{\mu}_{i, j}\right),
$$

where $i$ is the ID of reference regions, i.e., the ID of tissue type, with a range of $[0, N-1] ; N$, the number of reference regions; $j$, the pixel ID in the reference region, with a range of $\left[0, M_{i}-1\right]$; $M_{i}$, the number of pixels in the $i$-th reference region; $\mathbf{x}=\left[x_{0} \cdots x_{k} \cdots x_{M-1}\right]$, coordinate in the $M$-dimensional feature space; $k$, ID of the features used for the discrimination, with a range of $[0, L-1] ; L$, the number of features; $\boldsymbol{\mu}_{i, j}=\left[\mu_{i, j, 0}, \cdots, \mu_{i, j, k}, \cdots, \mu_{i, j, L-1}\right]$, coordinate of the $j$-th pixel of the $i$-th reference region in the feature space; $\mu_{i, j, k}$, value of the $k$-th feature of the $j$-th pixel in the $i$-th reference region. $G(\mathbf{x})$ is a Gaussian kernel in the feature space and is defined as

$$
G(\mathbf{x})=\exp \left[-\frac{1}{2} \sum_{k=0}^{L-1}\left(\frac{x_{k}}{\sigma_{k}}\right)^{2}\right]=\exp \left[-\frac{1}{2} \frac{\mathbf{x} \cdot \mathbf{x}^{T}}{\boldsymbol{\sigma} \cdot \boldsymbol{\sigma}^{T}}\right],
$$

where $\boldsymbol{\sigma}$ determines the kernel size, which is defined as

$$
\boldsymbol{\sigma} \equiv\left[\sigma_{0} \cdots \sigma_{k} \cdots \sigma_{L-1}\right]
$$


$\sigma_{k}$ is the kernel size for the $k$-th feature.

Finally, the similarity distribution is written as

$$
D_{i}(\mathbf{x})=\sum_{j=0}^{M_{i}-1} \exp \left[-\frac{1}{2} \sum_{k=0}^{L-1}\left(\frac{x_{k}-\mu_{i, j, k}}{\sigma_{k}}\right)^{2}\right]=\sum_{j=0}^{M_{i}-1} \exp \left[-\frac{1}{2} \frac{\left(\mathbf{x}-\boldsymbol{\mu}_{i, j}\right)\left(\mathbf{x}-\boldsymbol{\mu}_{i, j}\right)^{T}}{\boldsymbol{\sigma} \cdot \boldsymbol{\sigma}^{T}}\right] .
$$

A similarity distribution vector is defined as

$$
\mathbf{D}(\mathbf{x}) \equiv\left[D_{0}(\mathbf{x}) \cdots D_{i}(\mathbf{x}) \cdots D_{N-1}(\mathbf{x})\right]
$$

The similarity of the $p$-th pixel $\left(\mathbf{S}_{p}\right)$ to each reference region is then given as

$$
\mathbf{S}_{p} \equiv \mathbf{D}\left(\boldsymbol{\mu}_{p}\right)=\left[D_{0}\left(\boldsymbol{\mu}_{p}\right) \cdots D_{i}\left(\boldsymbol{\mu}_{p}\right) \cdots D_{N-1}\left(\boldsymbol{\mu}_{p}\right)\right]
$$

where $\mathbf{S}_{p}$ is an $N$-dimensional vector.

\subsection{Display of the discrimination results}

The similarity vector $\mathbf{S}_{p}$ is then converted to red-green-blue values for the purpose of display.

The typical color of the $i$-th reference region of the $i$-th tissue type is arbitrarily determined and expressed as

$$
\mathbf{C}_{i}=\left[R_{i} G_{i} B_{i}\right],
$$

where $R_{i}, G_{i}$, and $B_{i}$ are the red, green, and blue values of a pure $i$-th tissue. Namely, the respective color appears at a pixel that has the following similarity vector.

$$
\mathbf{S}_{p}=\left[S_{0} \cdots S_{a} \cdots S_{N-1}\right], \quad \text { where } S_{a}=\left\{\begin{array}{l}
1: a=i \\
0: a \neq i
\end{array} .\right.
$$

According to this similarity vector, the color conversion matrix is defined as

$$
\mathbf{A} \equiv\left[\mathbf{C}_{0}^{T} \cdots \mathbf{C}_{i}^{T} \cdots \mathbf{C}_{N-1}^{T}\right]=\left[\begin{array}{ccccc}
R_{0} & \cdots & R_{i} & \cdots & R_{N-1} \\
G_{0} & \cdots & G_{i} & \cdots & G_{N-1} \\
B_{0} & \cdots & B_{i} & \cdots & B_{N-1}
\end{array}\right]
$$

This matrix is a $3 \times N$ matrix.

Finally, the RGB values of the $p$-th pixel in a discriminated OCT image is calculated as follows.

$$
\mathbf{Q}_{p}=\mathbf{A} \mathbf{S}_{p}^{T} .
$$

Although we already have a discriminated OCT image $\mathbf{Q}$, yet another visualization of the composition of a log-scale intensity OCT and the discriminated OCT, which is referred to as a pseudo-color structural OCT, may be useful. The RGB value of the $p$-th pixel in the pseudocolor structural OCT is defined as follows.

$$
\mathbf{R}_{p} \equiv \frac{\mathbf{Q}_{p}}{\mathbf{L} \cdot \mathbf{Q}_{p}^{T}} I_{p}
$$

where $I_{p}$ is a logarithmically scaled OCT intensity of the $p$-th pixel. $\mathbf{L}$ is a luma matrix

$$
\mathbf{L} \equiv\left[\begin{array}{lll}
0.299 & 0.587 & 0.114
\end{array}\right]
$$

$\mathbf{L} \cdot \mathbf{Q}_{p}^{T}$ is a luma of the discriminated OCT $\mathbf{Q}_{p}$. 


\subsection{D tissue discrimination}

We created a 3D volume consisting of 256 pseudo-color structural OCT images. These B-scans were discriminated by a single similarity distribution. Because of B-scan dependent intensity difference, feature values slightly vary between different B-scans. Hence, we selected 2 Bscans that have a larger difference in intensity and summed up the similarity distribution vectors created from these $2 \mathrm{~B}$-scans. This summation is used as a unified similarity distribution vector for the discrimination of all B-scans of the volume. Namely, the similarity distribution is created only once for a $3 \mathrm{D}$ volume.

\section{Results}

Figure 1(a) shows images of the selected reference regions; the conjunctiva is shown in light brown; the sclera, in green; the TM, in dark yellow; cornea, in blue; and the uvea, in red. The pixels in the reference regions were then plotted in a 3D feature space of the intensity, extinction coefficient, and birefringence as shown in Fig. 1(b). The plotted colors in Fig. 1(b) are identical to the colors of the corresponding reference regions in Fig. 1(a). Feature distributions of the 5 reference regions were well separated in the 3D feature space. This indicates that the 3 parameters we used could successfully reflect the features of the target tissues.

The similarity distribution vector was calculated, and the final pseudo-color structural OCT image was created according to the method described above. We applied our method to 4 eyes of 4 subjects and similar discrimination results were obtained for all the eyes. Reference regions were selected for each subject. OCT images and discriminated results are shown in Fig. 2(a)-(d) and Fig. 2(e)-(h), respectively. We could observe different tissues with good contrast. The trabecular meshwork could be observed in the pseudo-color structural OCT image. The conjunctiva and sclera, which cannot be distinguished in conventional OCT images, could be clearly differentiated. However, although it is clinically not a significant error, a part of the cornea was discriminated as the uvea. This may because the selected reference region of uvea does not fully represent the property of the entire uveal tissues. Additional features, e.g., optic axis orientations and degree-of-polarization uniformity, and the higher dimensional feature space that results from them might improve the specificity of tissue discrimination. Black regions in the pseudo-color structural OCT image represent no similarities to any reference regions. The borders and outliers are apt to become black due to the limit of our current algorithm.

A TM line as observed by gonioscopy can be observed in the 3D volume rendered pseudocolor structural OCT. Fig. 3(a) shows a gonioscopic image and corresponding view can be available from 3D volume as shown in Fig. 3(b) and (c). The TM line is observed between the cornea and uvea as indicated by yellow arrows. The pseudo-color structural OCT volumes of a normal eye (Fig. 3(b)) and a narrow angle eye (Fig. 3(c)) can be clearly differentiated. According to the distance between the TM and the surface of the iris, we can intuitively assess the angle structures. Our method is useful for investigating the narrow angle eye, and it might be useful for the screening of patients for angle-closure glaucoma. Because OCT is a non-contact measurement method, we can apply this method as a non-contact alternative to gonioscopy. This OCT-based non-contact gonioscopic investigation can be easily applied for studying a large number of patient for large-scale screening, and can be applied to all patients, including who have undergone surgery and are hence at a high risk of infections.

\section{Discussion}

\subsection{Comparison with histological analysis}

We validated our method by applying our algorithm and histological data to fresh in vitro porcine eyes. For this measurement, we injected a marker into sub-Tenon's space. It was in- 


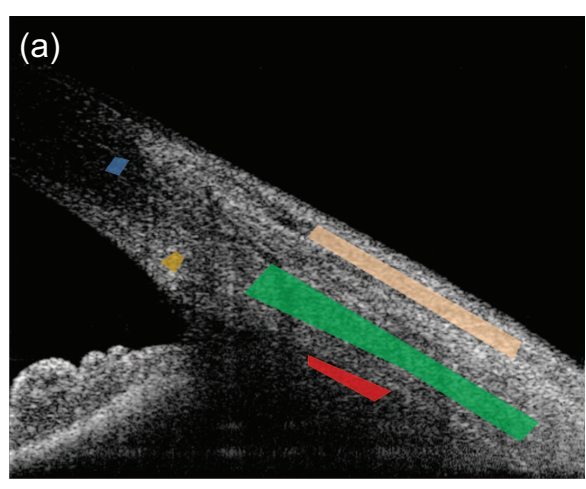

(b)

Extinction 75 coefficient $[\mathrm{dB} / \mathrm{mm}]$
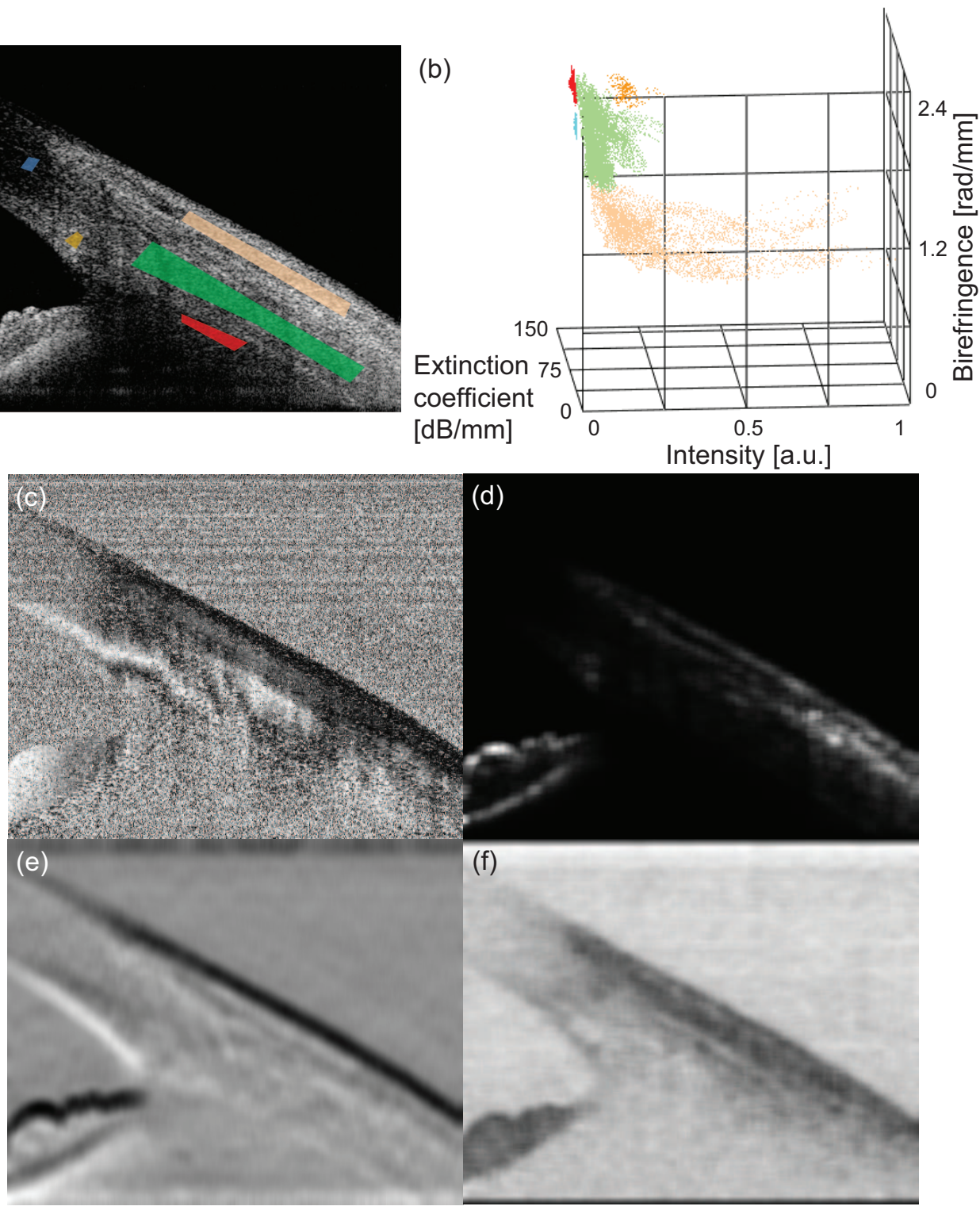

Fig. 1. (a) The 5 reference regions (light brown, conjunctiva; green, sclera; dark yellow, TM; blue, cornea; red, uvea) studied in subject 1 . They were selected manually on the basis of their anatomical and histological features; (b) Feature distributions of the 5 reference regions in the 3D (I-EC-BR) feature space. The corresponding phase retardation image (c), the OCT intensity in a linear scale (d), the extinction coefficient (e), and the birefringence (f) are also shown. 


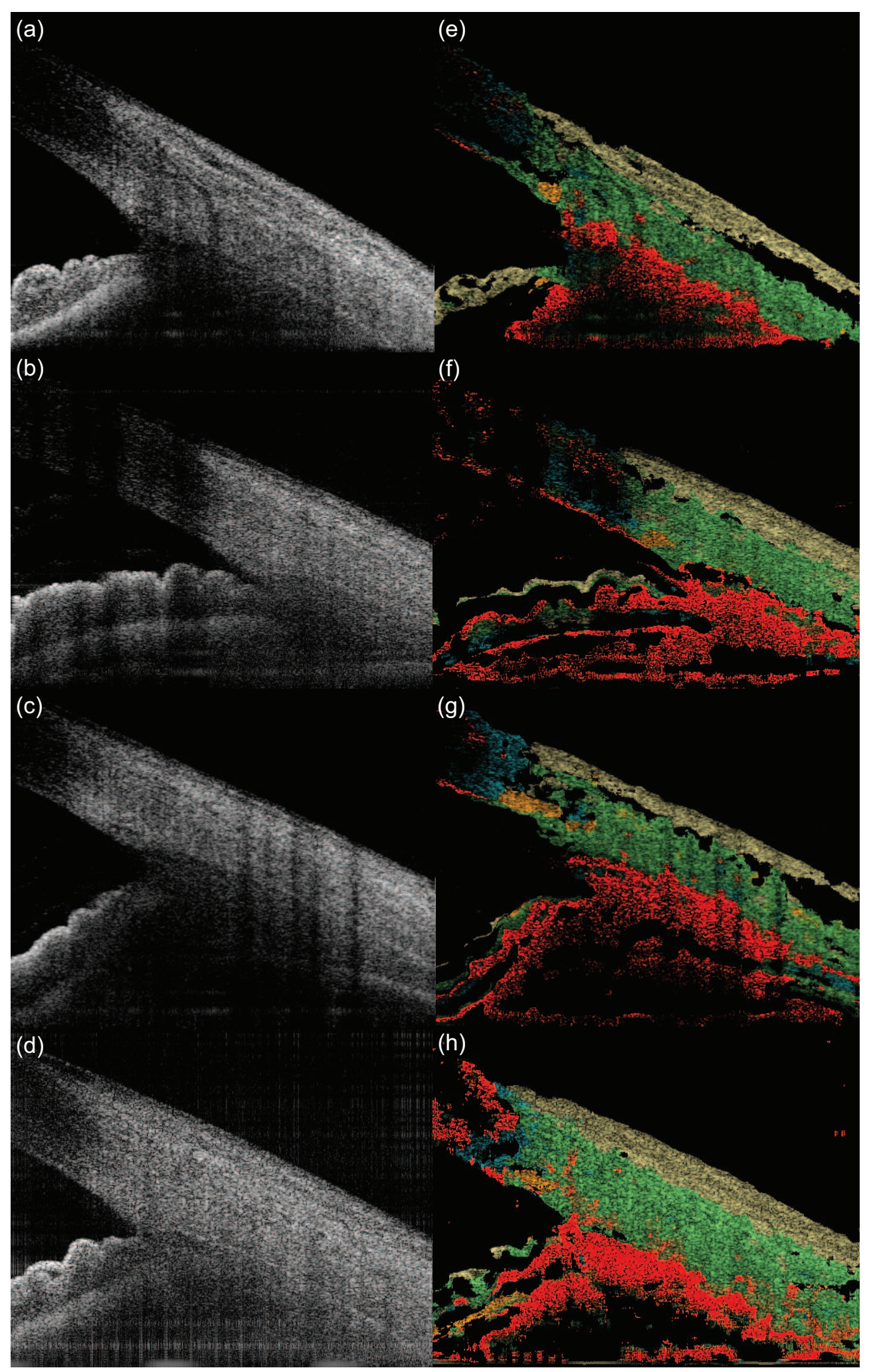

Fig. 2. Results of the tissue discrimination. First column - OCT images of subject 1(a), 2(b), 3(c), and 4(d); second column - discriminated results of subject 1(e), 2(f), 3(g), and 4(h) displayed as a pseudo-color structural OCT. The light brown indicates conjunctiva, green indicates sclera, dark yellow indicates TM, blue indicates cornea, and red indicates uvea. Different tissues can be observed with good contrast and the trabecular meshwork can also be seen.

\#115206 - \$15.00 USD Received 3 Aug 2009; revised 1 Sep 2009; accepted 5 Sep 2009; published 15 Sep 2009 (C) 2009 OSA 28 September 2009 / Vol. 17, No. 20 / OPTICS EXPRESS 17434 


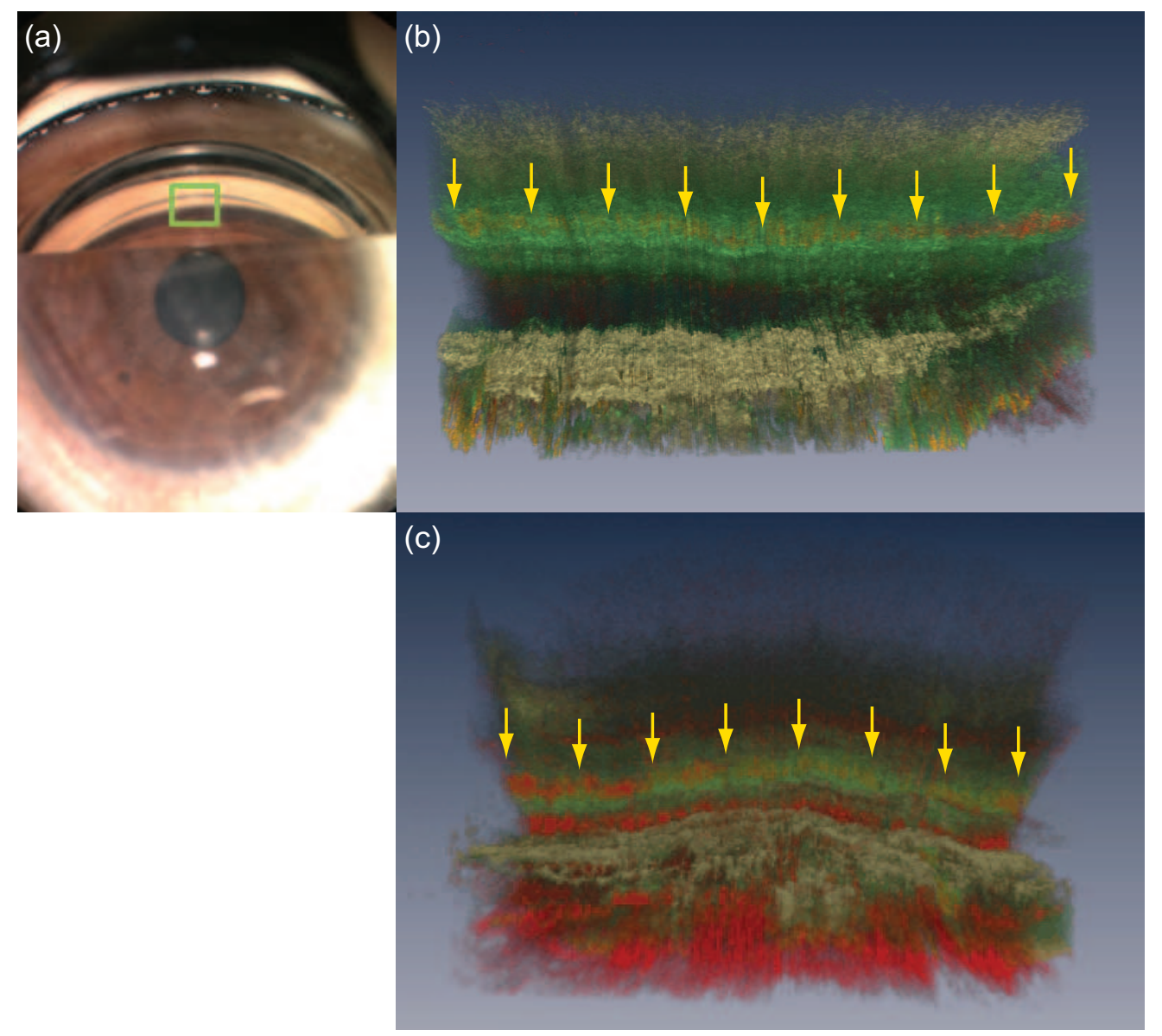

Fig. 3. (a) Gonioscopic image of the right eye of subject 1 at 9 o'clock position. The image is rotated clockwise by 90 degrees. 3-D discriminated volume of the (b) normal eye (Media 1) and (Media 2) and (c) narrow angle eye (Media 3) and (Media 4). These images correspond to the view of the green square in (a). TM can be observed as a yellow line as indicated by yellow arrows. 


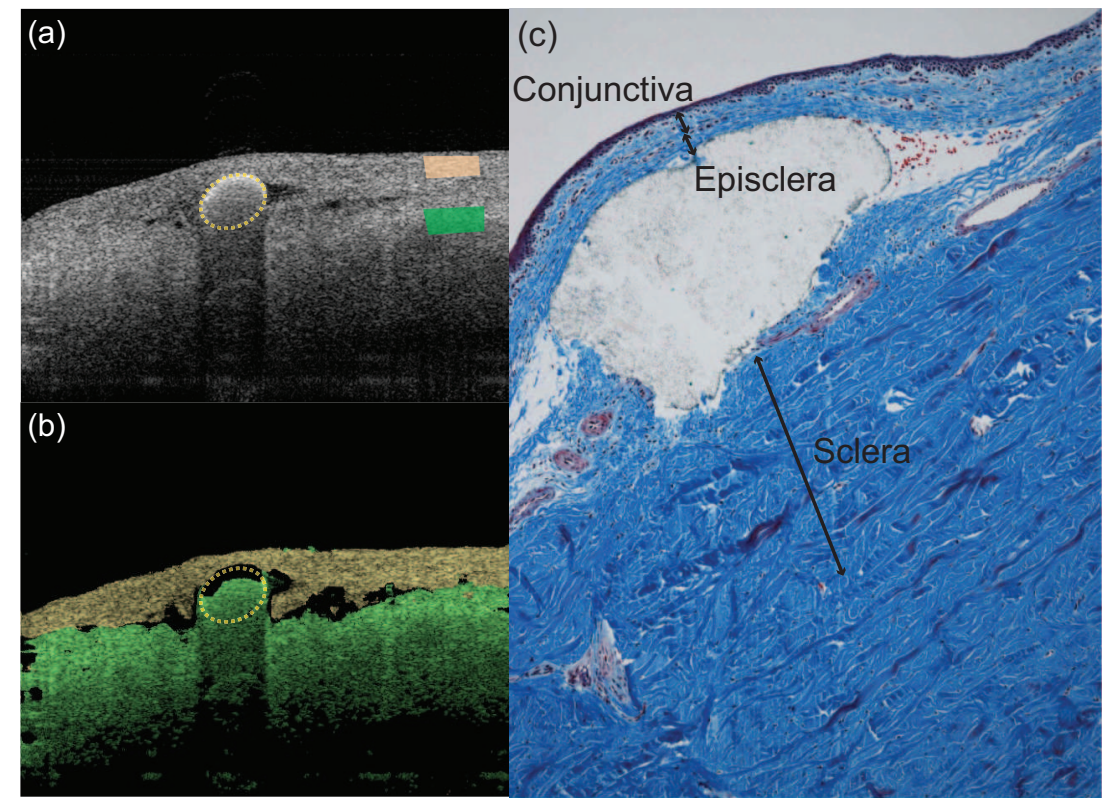

Fig. 4. (a) Intensity image of the porcine eye. The marker (dashed line) was injected into sub-Tenon's space. The reference regions for the conjunctiva (light brown) and sclera (green) were selected manually. (b) Pseudo-color structural OCT. Tissues that were anterior to the marker (dashed line) and posterior to the marker were segmented. (c) Specimen of the porcine eye stained by Masson's trichrome method. The marker was injected into subTenon's space. The anterior portion of the marker consists of the conjunctiva and episclera, and the posterior portion is identified as the sclera histologically.

jected with a $25 \mathrm{G}$ needle by an ophthalmologist. The marker injected was a thermoplastic resin adhesive (solvent type, product number 195, CEMEDINE CO. LTD.). We examined the site of injection by PS-OCT and applied the discrimination algorithm. A representative pseudocolor structural OCT is presented in Fig. 4. OCT image and discriminated result are shown in Fig. 4(a) and (b), respectively. For this discrimination, we used 2 reference regions, which were selected from the conjunctiva and sclera. We found that the selection of these reference regions results in the discrimination of tissues that are anterior to the marker and posterior to the marker. The tissue was processed to formalin-fixed and paraffin-embedded block, and cut into $5 \mu \mathrm{m}$-specimen. The specimen was stained by Masson's trichrome method, which stains collagen fiber blue, as shown in Fig. 4(c). According to the interpretations by a clinical ophthalmologist, an ophthalmic pathologist, and a pathology specialist, the marker was considered to be present in the region between the episclera and sclera. The episclera is the outermost layer of the sclera, which is a loose, highly vascular connective tissue, and is attached to Tenon's capsule. From this measurement, the region recognized as the conjunctiva according to our algorithm seems to include the conjunctiva itself, Tenon's capsule, and the episclera.

\subsection{Advantage of $3 D$ feature spaces}

The feature distributions were well separated in 3D feature spaces. However, they could not be separated by 1D or 2D analysis. Feature distributions in 2D feature spaces are shown in Fig. 5. In the I-EC space, the TM overlapped the sclera and conjunctiva (Fig. 5(a)). This suggests that birefringence obtained by PS-OCT played a decisive role in identifying the TM. In the EC- 
(a)

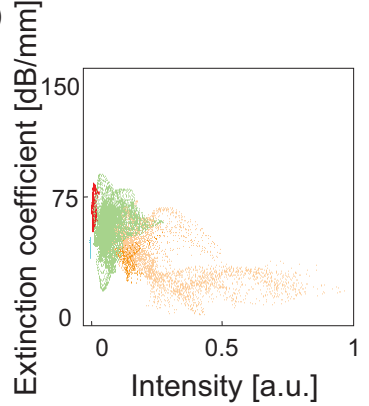

(b)

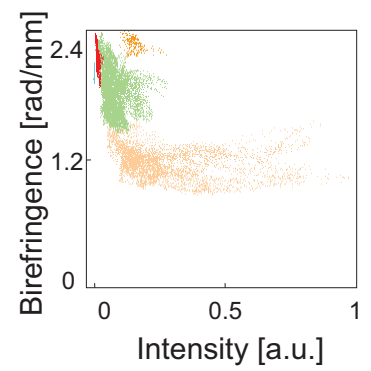

(c)

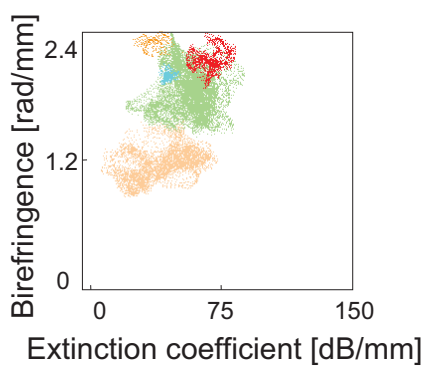

Fig. 5. Feature distributions in the 2D feature spaces (a) I-EC, (b) I-BR, and (c) EC-BR (light brown, conjunctiva; green, sclera; dark yellow, TM; blue, cornea; red, uvea). The 5 tissues could not be simultaneously discriminated by $1 \mathrm{D}$ or $2 \mathrm{D}$ analysis.

BR space, it is observed that the extinction coefficient can effectively be used to differentiate between the cornea and uvea (Fig. 5(c)).

The separation among the reference regions are quantified by using their normalized distances. The normalized distance between the distributions of $l$-th and $m$-th reference regions in respect to $n$-th feature is defined as

$$
\Delta_{l, m}^{(n)} \equiv \frac{\left|\mathbf{M}_{l}^{(n)}-\mathbf{M}_{m}^{(n)}\right|}{\sqrt{\left(\mathrm{SD}_{l}^{(n)}\right)^{2}+\left(\mathrm{SD}_{m}^{(n)}\right)^{2}}}
$$

where $\mathrm{M}_{l}^{(n)}$ and $\mathrm{SD}_{l}^{(n)}$ are the mean and the standard deviation of the distribution of $l$-th reference region in respect to $n$-th feature, respectively. $\mathrm{M}_{m}^{(n)}$ and $\mathrm{SD}_{m}^{(n)}$ are those of the $m$-th reference region. Subsequently, the normalized distance between the $l$-th and $m$-th reference regions in the 2D feature space of the $n$-th and $o$-th features is defined as

$$
\Delta_{l, m}^{(n, o)} \equiv \sqrt{\left(\Delta_{l, m}^{(n)}\right)^{2}+\left(\Delta_{l, m}^{(o)}\right)^{2}},
$$

and that in the 3D feature space of the $n$-th, $o$-th and $p$-th features is defined as

$$
\Delta_{l, m}^{(n, o, p)} \equiv \sqrt{\left(\Delta_{l, m}^{(n)}\right)^{2}+\left(\Delta_{l, m}^{(o)}\right)^{2}+\left(\Delta_{l, m}^{(p)}\right)^{2}} .
$$

The normalized distances were obtained for all pairs of 5 reference regions in each 1D to 3D feature space as shown in Fig. 6, where the same dataset and the same reference regions with Fig. 1(a) were used. The horizontal axes represent the combination of the features and the vertical axes represent the normalized distance. The averaged normalized distance of all the pairs of reference regions was improved to be 7.14 in 3D feature space, while those in $1 \mathrm{D}$ feature spaces were 3.12, 2.03 and 5.15 for I, EC, and BR, respectively, and those in 2D feature spaces were 6.62 for I-BR, 3.92 for I-EC, and 5.97 for EC-BR. This analysis quantitatively indicates that the usage of a larger number of features provides better separation of reference regions. It is also noteworthy that all distances, except of the TM-uvea and the sclera-cornea, increased when the birefringence were involved as shown in Figs. 6(a)-(d). We also find that the introduction of extinction coefficient increases the distance between cornea and uvea effectively, as shown in Figs. 6(a)-(b) and 6(e)-(f). 
(a)

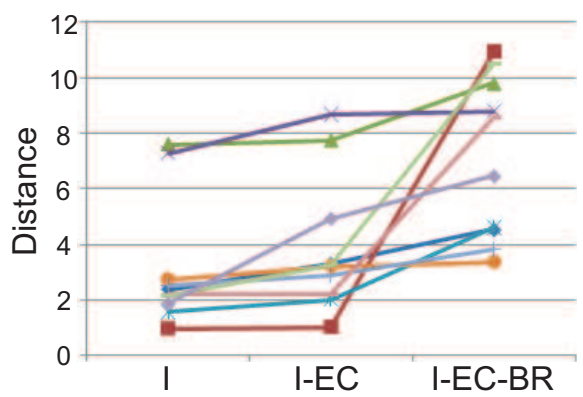

(c)

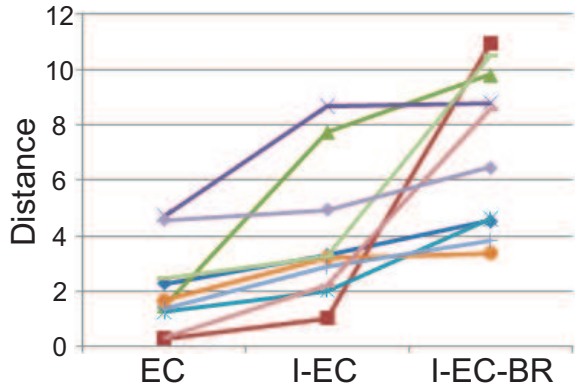

(e)

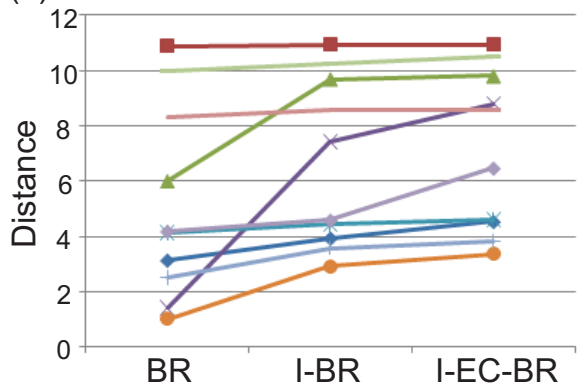

(b)

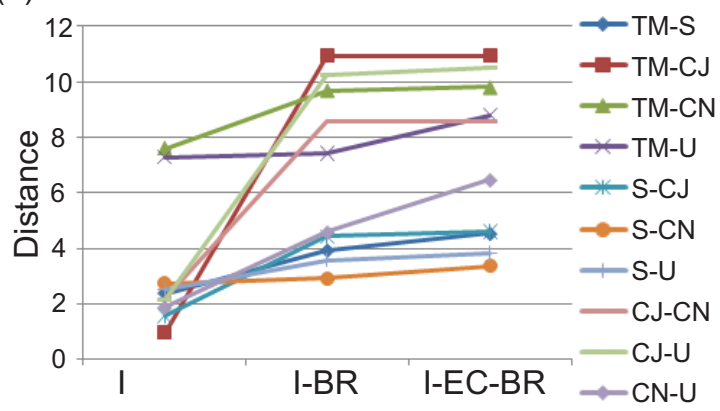

(d)

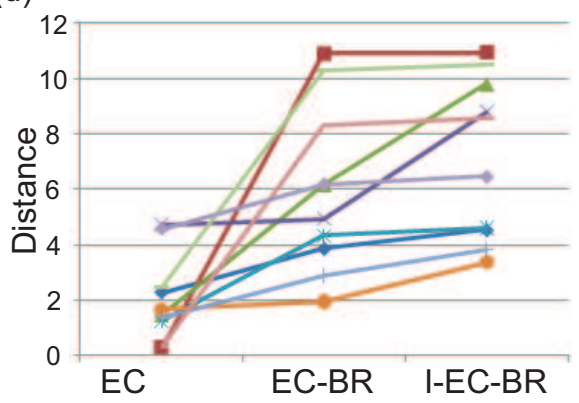

(f)

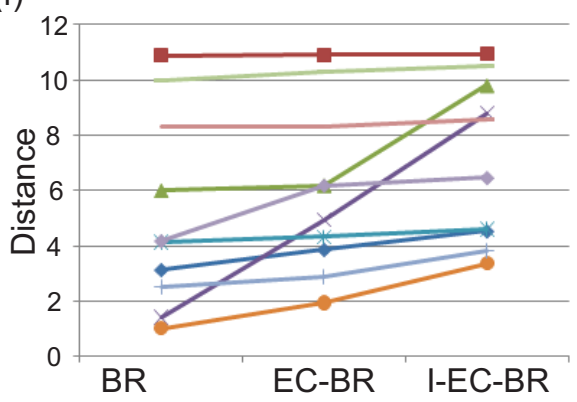

Fig. 6. The normalized distances of all the pairs of the 5 reference regions in each feature space. The same dataset and the same reference regions with those of Fig. 1(a) were used. I: intensity, EC: extinction coefficient, BR: birefringence; TM: trabecular meshwork, S: sclera, CJ: conjunctiva, $\mathrm{CN}$ : cornea, and U: uvea. 


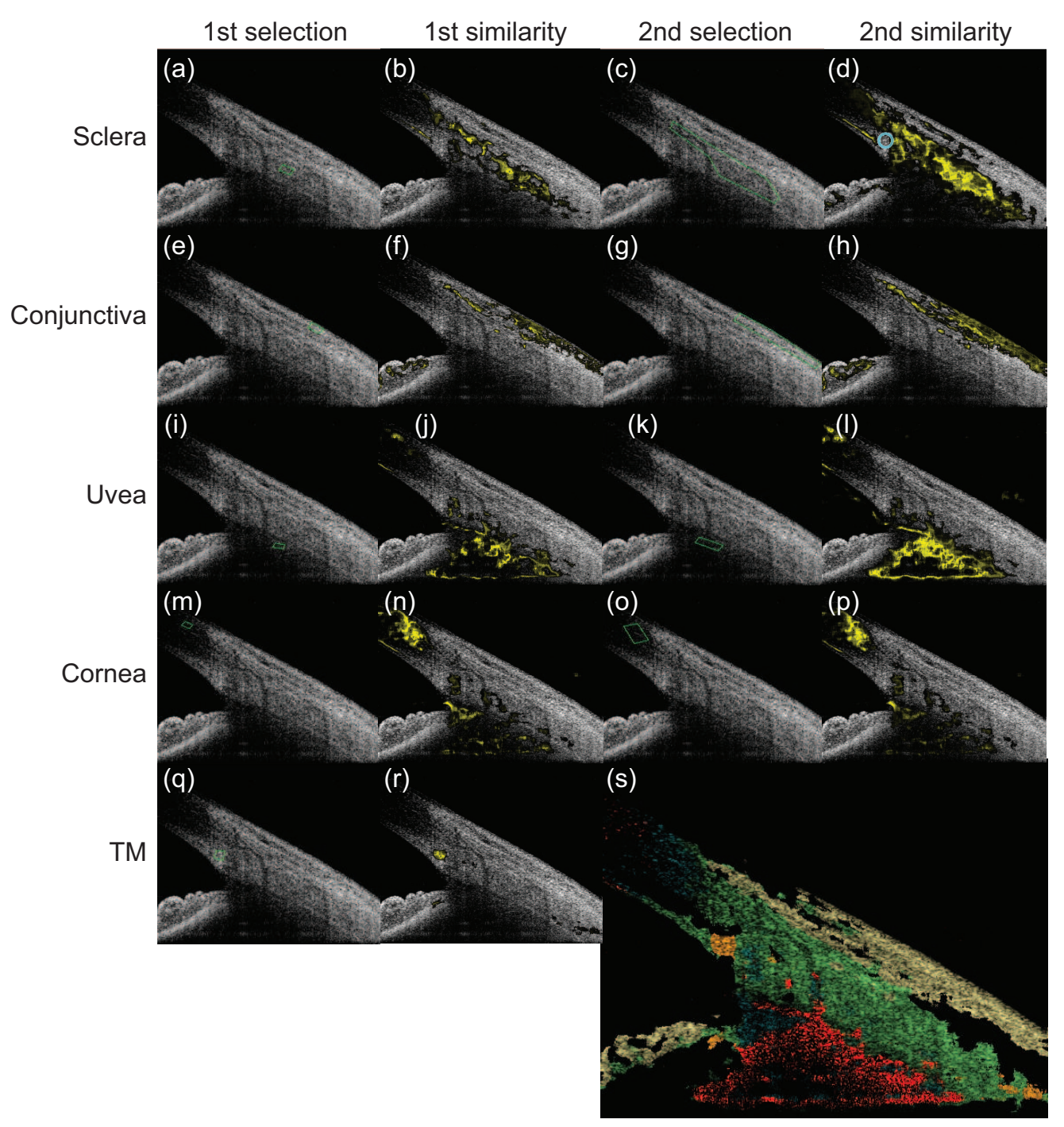

Fig. 7. (a)-(r) An example of the selection of reference regions. The raw represents the tissue of interest. The first and second columns represent the reference regions selected in the first step, and similarities obtained from the first selected reference regions, respectively. The third and fourth columns represent the reference regions of the second, i.e. the final, step, and similarities obtained from this final selections. (s) A pseudo-color structural OCT image obtained by the reference regions indicated in (c), (g), (k), (o) and (q).

\subsection{Selection of the reference regions}

In our current algorithm, the reference regions are manually selected. To make the result robust to the fluctuation of the selection of the reference regions, we employed the following maneuver.

First, except for the selection of TM, we selected a small reference region which was surely a part of the tissue of interest as shown in Figs. 7(a), (e), (i) and (m). Second, the operator monitored the similarity distribution obtained with the first small reference region as shown in the Figs. 7(b), (f), (j) and (n) (yellow regions). The operator then selected the second reference 
region to include the high similarity regions of the first similarity map. And this second reference region was used for the subsequent tissue discrimination analysis. This two-step selection protocol made our algorithm robust.

The reference region of TM was selected after the selection of the sclera. The similarity of the sclera showed a void region close to the scleral spur as indicated by a blue circle in Fig. 7(d). This region was then selected as the reference region of TM as shown in Fig. 7(q).

Figure 7(s) shows the final pseudo-color structural OCT image. This image and the image shown in Fig. 2(e) were created from the same dataset, but the reference regions were selected independently by a single operator with a time interval of one month. It is noteworthy that these two images show similar results despite the reference regions have been selected independently.

The automation of this selection procedure may increase the stability of the algorithm, and this may be a subject of a future development.

\subsection{Error in BR measurement}

In the Fig. 1(b), conjunctiva shows higher birefringence than expected. This relatively high birefringence is an artifact and comes from asymmetric distribution of BR in the feature space.

Our PS-OCT algorithm is based on Jones matrix formalism and the range of phase retardation as well as BR is limited from 0 to 180 degrees. If the true BR is close to zero, as in the case of conjunctiva, additional noises shift some of BR values to the negative range, and then this negative $\mathrm{BR}$ value is aliased into the above mentioned positive range of 0 to 180 degrees [33, 35, 36]. This aliasing skews the distribution of the measured BR. Consequently, the window averaging of this skewed distribution provides a slightly up-shifted BR value. A similar and detailed discussion is described in Ref. 37.

\section{Conclusion}

We developed a tissue discrimination algorithm using 3 parameters of optical properties obtained by PS-OCT. This discrimination algorithm is not based on structural information, but directly on the properties of the tissues. The conjunctiva, sclera, TM, cornea, and uvea were well separated in the 3D feature space and could be successfully discriminated. In the 3D discriminated volumes, the TM line was clearly observed. Thus, the combination of 3 parameters enabled simultaneous discrimination of the 5 tissues. In particular, the birefringence obtained by PS-OCT played an important role in the discrimination of the TM.

\section{Acknowledgement}

This study is partially supported by the Japan Society for the Promotion of Science (JSPS) through the Grant-in-aid for Scientific Research 15760026, and the Japan Science and Technology Agency through the contract of the development program of advanced measurement systems. 